\title{
3D CFD computations of transitional flows using DES and a correlation based transition model
}

Sørensen, Niels $\mathbf{N}$.

Publication date:

2009

Document Version

Publisher's PDF, also known as Version of record

Link back to DTU Orbit

Citation (APA):

Sørensen, N. N. (2009). 3D CFD computations of transitional flows using DES and a correlation based transition model. Danmarks Tekniske Universitet, Risø Nationallaboratoriet for Bæredygtig Energi. Denmark.

Forskningscenter Risoe. Risoe-R No. 1692(EN)

\section{General rights}

Copyright and moral rights for the publications made accessible in the public portal are retained by the authors and/or other copyright owners and it is a condition of accessing publications that users recognise and abide by the legal requirements associated with these rights.

- Users may download and print one copy of any publication from the public portal for the purpose of private study or research.

- You may not further distribute the material or use it for any profit-making activity or commercial gain

- You may freely distribute the URL identifying the publication in the public portal

If you believe that this document breaches copyright please contact us providing details, and we will remove access to the work immediately and investigate your claim 
3D CFD computations of transitional flows using DES and a correlation based transition model

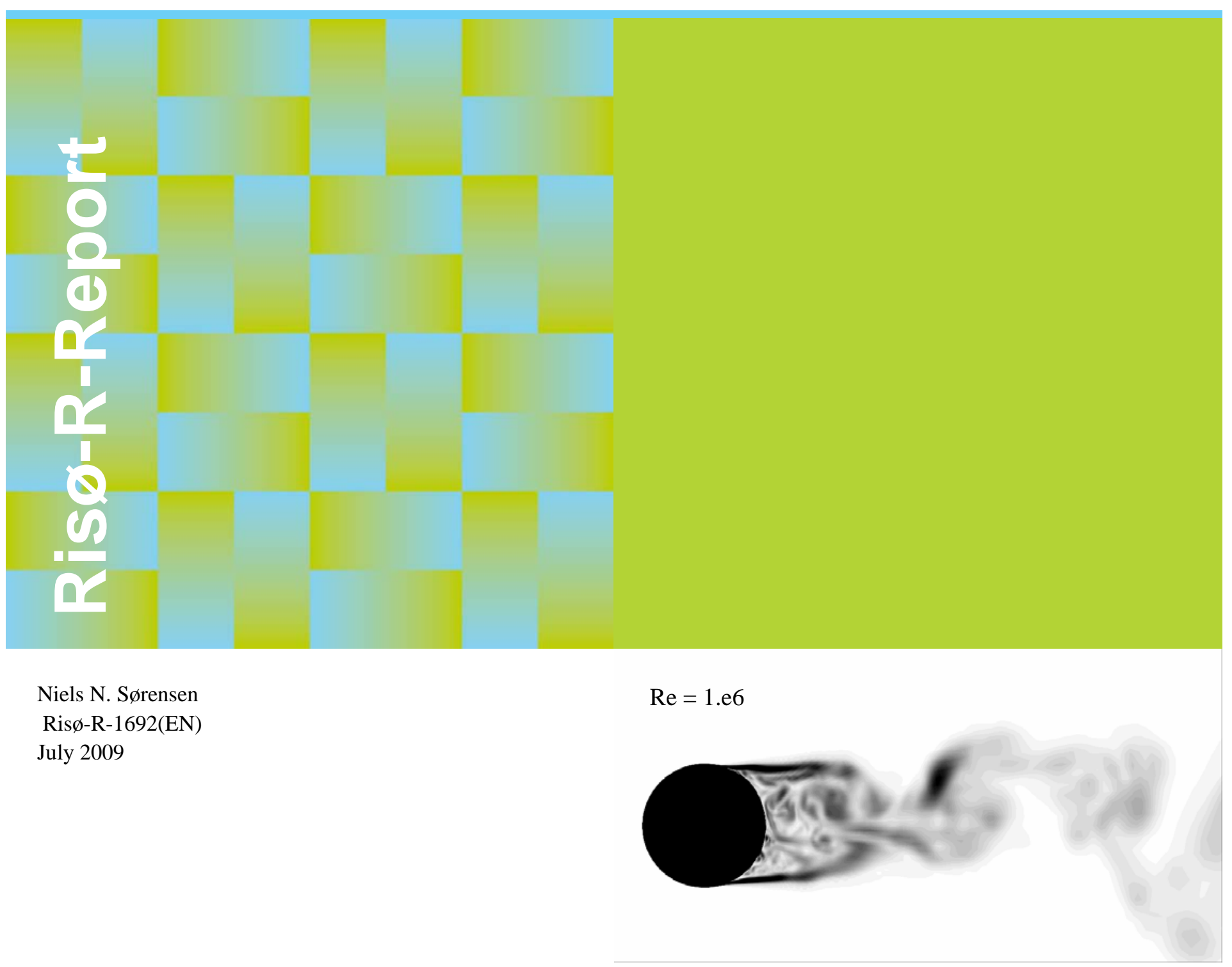


Title: 3D CFD computations of transitional flows using DES and a correlation based transition model

Department: Aeroelastic Design - Wind Energy Division

\section{Abstract:}

The report describes the application of the correlation based transition model of of Menter et. al. [1, 2] to the cylinder drag crisis and the stalled flow over an DU-96-W-351 airfoil using the DES methodology. When predicting the flow over airfoils and rotors, the laminar-turbulent transition process can be important for the aerodynamic performance. Today, the most widespread approach is to use fully turbulent computations, where the transitional process is ignored and the entire boundary layer on the wings or airfoils is handled by the turbulence model. The correlation based transition model has lately shown promising results, and the present paper describes the application of the model to predict the drag and shedding frequency for flow around a cylinder from sub to super-critical Reynolds numbers. Additionally, the model is applied to the flow around the DU-96 airfoil, at high angles of attack.
ISSN

ISBN 978-87-550-3749-6

\section{Contract no.:}

ENS-33033-0055

Group's own reg. no.:

Sponsorship:

Danish Energy Agency

Cover:

Separation behind cylinder

Pages: 18

Tables: 17

References: 24

Information Service Department

Ris $\varnothing$ National Laboratory for Sustain-

able Energy

Technical University of Denmark

P.O.Box 49

DK-4000 Roskilde

Denmark

Telephone +45 46774004

bibl@risoe.dk

Fax +4546774013

www.risoe.dk 


\section{Contents}

1 Abstract 3

\section{Introduction 3}

3 Code description 3

3.1 Transition Model 4

4 Results 5

4.1 Computational grids 5

4.2 Flow Over a Circular Cylinder 6

4.3 Discussion of Cylinder Results 9

4.4 Flow over a thick airfoil 10

4.5 Discussion of Airfoil Results 15

5 Conclusion 15

6 Acknowledgement 15 


\section{Abstract}

The present report describes the application of the correlation based transition model of of Menter et al. $[1,2]$ to the flow over a cylinder and a thick airfoils using the Detached Eddy Simulation (DES) methodology. When predicting the flow over airfoils and rotors, the laminarturbulent transition process can be important for the aerodynamic performance. Today, the most widespread approach is to use fully turbulent computations, where the transitional process is ignored and the entire boundary layer on the wings or airfoils is handled by the turbulence model. In the present work the possibility of combining the DES technique with a transition model is tested for two flows featuring large separated areas.

\section{Introduction}

During the last years, Computational Fluid Dynamics has found wide spread use within the wind energy community, and has been shown to perform well in many cases. Even though much success has been achieved, important problems still exist where the standard fully turbulent Reynold Averaged Navier-Stokes (RANS) approach fails to give sufficiently accurate answers. The most obvious problem is the failure to predict the power production and load for stall controlled turbines at high wind, corresponding to high angle of attack along the blade span. Attempting to shed some light on some of the fundamental problems connected to the deep stall physics of wind turbine blades, often equipped with thick airfoil, the present work firstly addresses the classical problem of predicting the drag crisis of a circular cylinder, secondly applying the same methodology to the flow over a thick airfoil. The cylinder case is mainly chosen because a large body of high quality experimental data exists, which for many other flows can be problematic to obtain for deep stall cases. Additionally, cylindrical or nearly cylindrical sections exist at the inboard part of most modern wind turbine rotors. To accomplish the flow simulations, the new correlation based $\gamma-R e_{\theta}$ model by Menter et al. [1] and the DES version of the $k-\omega$ SST model by Strelets [3] is applied. It is well known that the movement of the separation point on the circular cylinder is highly influenced by the laminar to turbulent transition process. Additionally it is well know that typical RANS are not sufficiently accurate in massively separated flows, and to help alleviate this problem, the DES technique is applied.

\section{Code description}

The in-house flow solver EllipSys3D is used in all computations presented in this paper. The code is developed in co-operation between the Department of Mechanical Engineering at the Technical University of Denmark and The Department of Wind Energy at Risø National Laboratory, see [4,5] and [6]. The EllipSys3D code is a multiblock finite volume discretization of the incompressible Reynolds Averaged Navier-Stokes (RANS) equations in general curvilinear coordinates. The code uses a collocated variable arrangement, and Rhie/Chow interpolation [7] is used to avoid odd/even pressure decoupling. As the code solves the incompressible flow equations, no equation of state exists for the pressure, and in the present work the Semi-Implicit Method for Pressure-Linked Equations (SIMPLE) algorithm of Patankar and Spalding [8, 9] or the Pressure Implicit with Splitting of Operators (PISO) algorithm of Issa $[10,11]$ is used to enforce the pressure/velocity coupling, for steady state and transient computations respectively. The EllipSys3D code is parallelized with the Message-Passing Interface (MPI) for executions on distributed memory machines, using a non-overlapping domain decomposition technique.

Both steady state and unsteady computations can be performed. For the unsteady computa- 
tions the solution is advanced in time using a 2nd order iterative time-stepping (or dual timestepping) method. In each global time-step the equations are solved in an iterative manner, using under relaxation. First, the momentum equations are used as a predictor to advance the solution in time. At this point in the computation the flowfield will not fulfil the continuity equation. The rewritten continuity equation (the so-called pressure correction equation) is used as a corrector making the predicted flowfield satisfy the continuity constraint. This two step procedure corresponds to a single sub-iteration, and the process is repeated until a convergent solution is obtained for the time step. When a convergent solution is obtained, the variables are updated, and we continue with the next time step. Thus, when the sub-iteration process is finished all terms are evaluated at the new time level.

For steady state computations, the global time-step is set to infinity and dual time stepping is not used, this corresponds to the use of local time stepping. In order to accelerate the overall algorithm, a multi-level grid sequence is used in the steady state computations. The convective terms are discretized using a third order Quadratic Upstream Interpolation for Convective Kinematics (QUICK) upwind scheme, implemented using the deferred correction approach first suggested by Khosla and Rubin [12]. Central differences are used for the viscous terms, in each sub-iteration only the normal terms are treated fully implicit, while the terms from non-orthogonality and the variable viscosity terms are treated explicitly.

In the present work the turbulence in the boundary layer is modeled by the k- $\omega$ Shear Stress Transport (SST) eddy viscosity model [13] using it as the DES model as proposed by Strelets [3], to account for the deep stall physics behind the cylinder. Due to problems often referred to Modeled Stress Depletion (MSD) reported for the standard DES method, when the resolved LES layer penetrates too deep into the RANS boundary layer, the DDES variation of the DES methodology proposed by Menter [14] using the f2zone as a shield function was tested. The problem of the LES region contaminating the RANS layer can happen when computing flows with high values of inflow turbulence typically used with the correlation based transition model. The equations for the turbulence model and the transition model are solved after the momentum and pressure correction equations in every sub-iteration/pseudo time step, and in agreement with the recommendations of Menter et al. [1], a second order upwind Total Variation Diminishing (TVD) scheme based on the MinMod limiter is used for the transport equations for turbulence and transition. The three momentum equations, the $k-\omega$ equations and the two transition model equations are solved decoupled using a red/black Gauss-Seidel point solver. The solution of the Poisson system arising from the pressure correction equation is accelerated using a multigrid method.

\subsection{Transition Model}

The $\gamma-\widetilde{\operatorname{Re}}_{\theta}$ correlation based transition model of Menter [1], is a framework for implementing empirical correlations based transition criterions in general purpose flow solvers, that can be used together with structured, unstructured and parallelized solvers. The backbone of the model is two transport equations one for intermittency $\gamma$ and one for the local transition onset momentum thickness Reynolds number $\widetilde{\operatorname{Re}}_{\theta t}$. Basically, the model relates the local momentum thickness Reynolds number $\operatorname{Re}_{\theta}$ to the critical value $\operatorname{Re}_{\theta c}$, and switches on the intermittency production when $\operatorname{Re}_{\theta}$ is larger than the local critical value. Based on a series of zero pressure gradient flat plate boundary layers, expressions for the two missing correlation functions relating $\operatorname{Re}_{\theta c}$ and $F_{\text {length }}$ to $\widetilde{\operatorname{Re}}_{\theta t}$ have been determined by Sørensen [15].The functional dependency of the two correlations are approximated by the following expressions:

$$
\operatorname{Re}_{\theta c}=\beta\left(\frac{\widetilde{\operatorname{Re}_{\theta t}}+12000}{25}\right)+(1-\beta)\left(\frac{7 \cdot \widetilde{\operatorname{Re}_{\theta t}}+100}{10}\right),
$$

and 


$$
F_{\text {length }}=\min \left[150 \cdot \exp \left[-\left(\frac{\widetilde{\operatorname{Re}}_{\theta t}}{120}\right)^{1.2}\right]+0.1,30\right] .
$$

where $\beta$ is a blending function defined as:

$$
\beta=\tanh \left[\left(\widetilde{\frac{\operatorname{Re}_{\theta t}}{400}-100}\right)^{4}\right] \text {. }
$$

Comparing the present correlation for $R e_{\theta c}$ with the two correlations proposed by Toyoda et al. [16] and Pettersson et al. [17] good agreement is observed at low $R e_{\theta t}$, see Figure 1. In the paper by Toyoda et al. the expression for the $F_{\text {length }}$ parameter has dimension of length, and not a dimensionless quantity as it should be. A direct comparison of the expression of Toyoda et al. and the present $F_{\text {length }}$ parameter therefore is not possible, whereas the correlation proposed by Pettersson et al. show good agreement, see Figure 1.
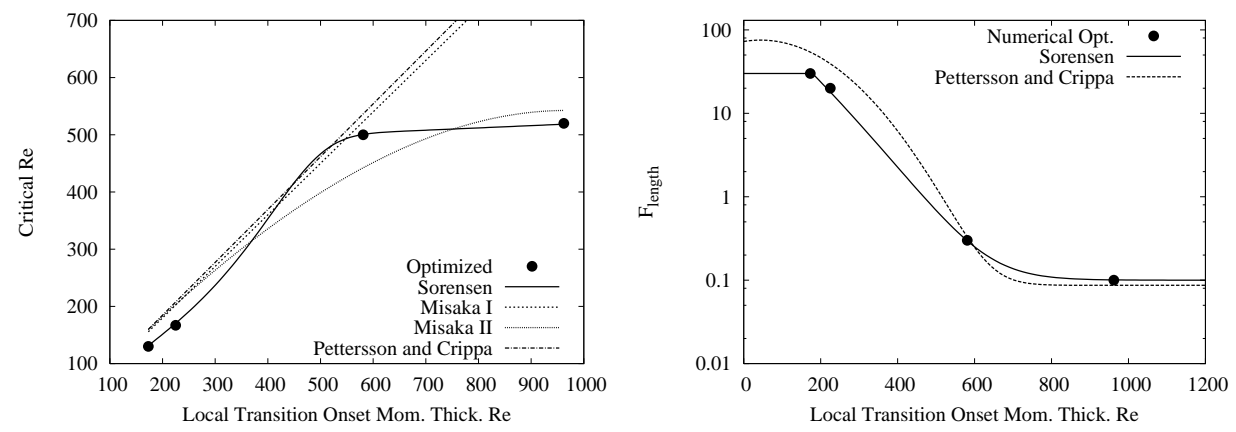

Figure 1. Comparison between the four target points from the optimization and different correlation functions.

It is well known that the turbulence will decay from the inlet value, in the case of zero shear where there is no production in the farfield. To control the level of turbulent kinetic energy at the boundary layer edge, the farfield value can be estimated from Eqn. 4, from [18]:

$$
k=k_{\text {inlet }}\left(1+\omega_{\text {inlet }} \beta t\right)^{-\frac{\beta^{*}}{\beta}},
$$

with $\beta=0.09$, and $\beta^{*}=0.828$.

\section{Results}

\subsection{Computational grids}

The meshes for the computations in the present work are generated with the $2 \mathrm{D}$ enhanced hyperbolic grid generation program HypGrid2D [19] as a 2D slice, and the 3D grid is then generated by sweeping the grid in the span-wise direction, see Figure 2. For the cylinder flow, the O-mesh has 256 cells around the surface of the cylinder, 256 cells in the normal direction with a first cell height $\left(\frac{\Delta z}{D}\right)$ of $1 \times 10^{-5}$ and the outer domain boundary placed approximately 30 diameters away from the surface. In the span-wise direction 128 cells are used along the $2 \times D$ span, giving a cell size of around $0.0156 \mathrm{D}$. The grid holds in total 8.4 million cells. 
The surface of the cylinder is modeled as a no-slip surface, and periodic conditions are used in the spanwise direction. The outer domain boundary is specified as inlet, except for the area downstream of the cylinder covering around +/- 45 degrees in azimuth direction, where outlet condition is used specifying fully developed flow.

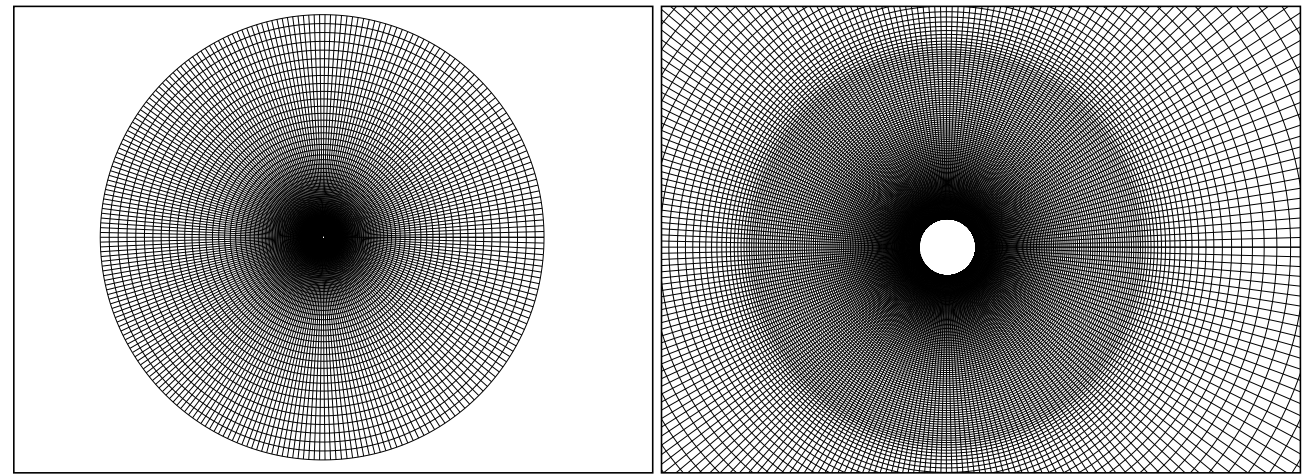

Figure 2. Mesh around the circular cylinder.

The O-mesh for the DU-96-W351 airfoil was generated with the HypGrid2D program, with 512 cells in the chordwise direction, 256 cells in the normal direction with a first cell height $\left(\frac{\Delta z}{C}\right)$ of $5 \times 10^{-6}$ and the outer domain boundary placed approximately 60 chords away from the surface, see Figure 3 . In the span-wise direction 128 cells are used along the $2 \times C$ span, giving a cell size of around $0.0156 \times C$. The grid holds in total 17 million grid points. The surface of the airfoil is modeled as a no-slip surface, and periodic conditions are used in the spanwise direction. The outer domain boundary is specified as inlet, except for the area downstream of the airfoil covering around $+/-45$ degrees in azimuth direction, where outlet condition is used specifying fully developed flow.

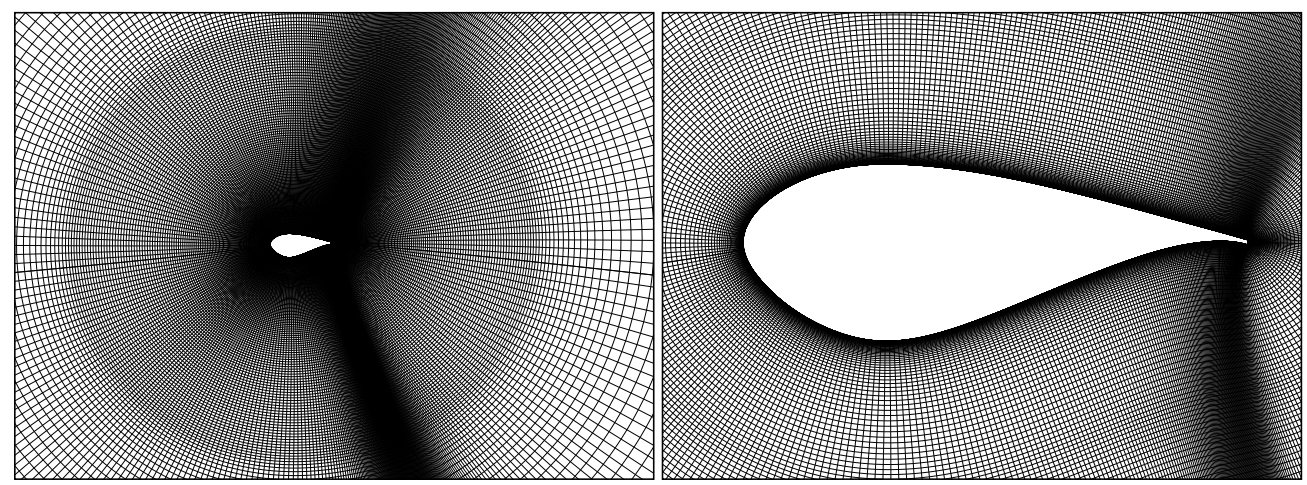

Figure 3. Mesh around the DU profile.

\subsection{Flow Over a Circular Cylinder}

The challenging case of flow over the cylinder covering the full range from laminar flow at low Reynolds numbers to transitional flow at high Reynolds numbers is investigated. The case is chosen to show how the transition model can account both for the pure laminar flow at low Reynolds numbers and the complex physics taking place around the drag crisis at the critical Reynolds number of the cylinder. From a practical point of view, the circular cylinder is often used on the inboard part of many modern wind turbine blades as well as for typical turbine 
towers.

The computations are performed at a time step of $\Delta t \cdot U / c=0.01$ using four sub-iterations and the PISO algorithm. Typical computing times for the 8.4 million grid points are approximately 16 seconds per time-step using 32 Dell PowerEdge 750 one processor machines, based on Intel Pentium 4 (Prescott) $3.2 \mathrm{GHz}$ processors with $1 \mathrm{MB}$ cache. Each machine has $2 \mathrm{~GB}$ of memory, the interconnect is based on Gigabit Ethernet network. To obtain reliable statistics the computations were typically performed for at least 200 to $300 \times U / D$, requiring around a week of computations on a 32 processors.

A typical time trace of the drag force is shown in Figure 4, where a strong variation of the drag at the startup of the computations is observed, and the more stochastic behavior in the 'periodic' state. To obtain reliable drag estimates, typically the first 50 dimensionless times are discarded and only the remaining part of the time series is used for analysis. This practice is used both in connection with the spectral analysis of the results as well as for determining the mean values.

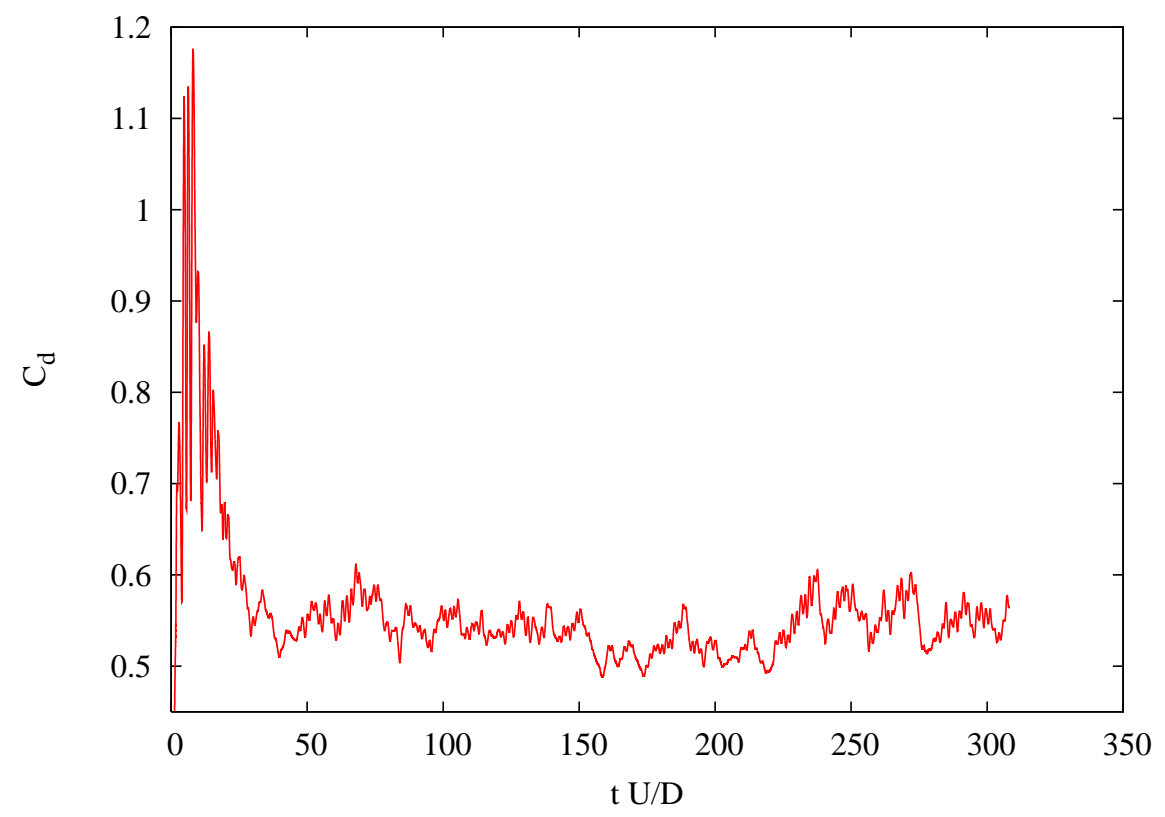

Figure 4. The time history of the drag force for the case of a Reynolds number of $5 \times 10^{5}$, showing the strong transient at the impulsive startup and the stochastic behavior in the 'periodic' state.

Comparing the computed drag $\left(\frac{F_{D}}{0.5 \mathrm{p} U^{2}}\right)$ as function of Reynolds Number with measured values [20], an improved agreement around the drag crisis is observed, see Figure 5, where the transitional computations predict the increase of the drag in the region of Reynolds number from $1 \times 10^{4}$ to $2 \times 10^{5}$ observed in the measurements. The fully turbulent computations, in contrast, predict a gradual decrease of drag underestimating the drag more than $30 \%$ in this region. Additionally, using the transition model the flow around the cylinder can be predicted with the same computational settings from a Reynolds number of 10 to $1 \times 10^{6}$ without any changes, with excellent results.

The Strouhal number $\left(\frac{f D}{U}\right)$ is determined from the power spectrum of the normal force, and an example of a power spectrum for a Reynolds number of $1 \times 10^{4}$ is shown in Figure 6 . The power spectrum indicates that the Strouhal frequency is clearly defined and most energy is concentrated at this frequency. Looking at the variation of the Strouhal number as function of Reynolds number shown in Table 1 and Figure 7, the typically reported value of 0.2 is found for the lower range of Reynolds numbers below $R e=1 \times 10^{5}$. In the range between $R e=1 \times 10^{5}$ 


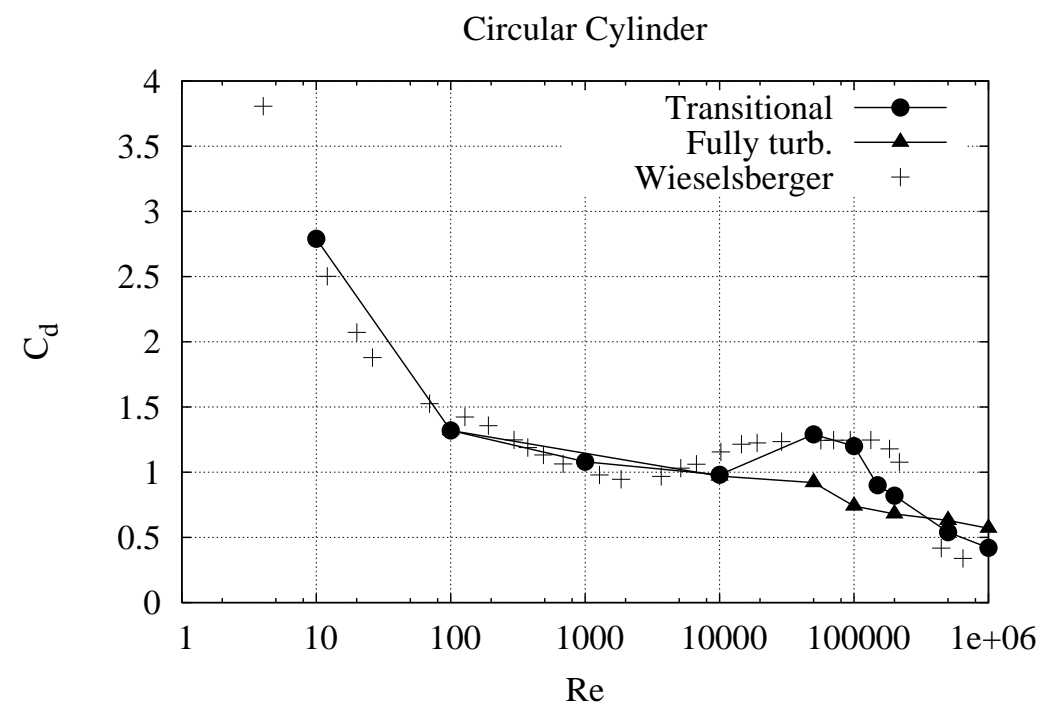

Figure 5. Comparison of computed drag force for the fully turbulent and the transitional computations with the measurements.

to $R e=1 \times 10^{6}$ the computations predict an increase of the Strouhal frequency, not normally discussed, but in fair agreement with several references [21, 22, 23]. In this range the Strouhal frequency increase more than $60 \%$, which for some applications may be of importance.

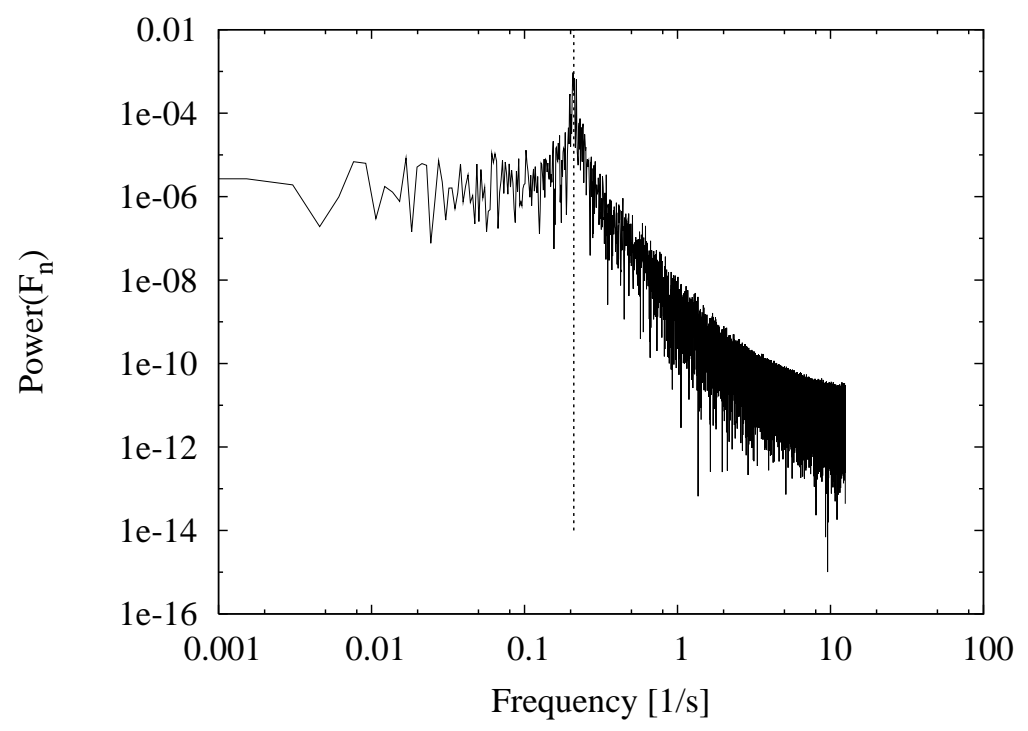

Figure 6. Identification of the Strouhal number for the Re=10000 case, using the power spectrum.

Observing the development of the limiting streamlines at the surface of the cylinder and the movement of the laminar to turbulent transition, insight about the actual physical process can be obtained. For the lowest Reynolds number computed, a steady solution without spanwise variation is obtained, with a small separation bubble at the aft part of the cylinder. Increasing the Reynolds number to 100 , a periodic solution with vortex shedding is obtained, still with zero spanwise variation. For a Reynolds number of $1 \times 10^{3}$, the wake is starting to be turbulent, and the flow is no longer fully correlated along the span of the cylinder. For a Reynolds number around $1 \times 10^{4}$, Figure 9 , the flow is laminar over the front part of the cylinder in good 


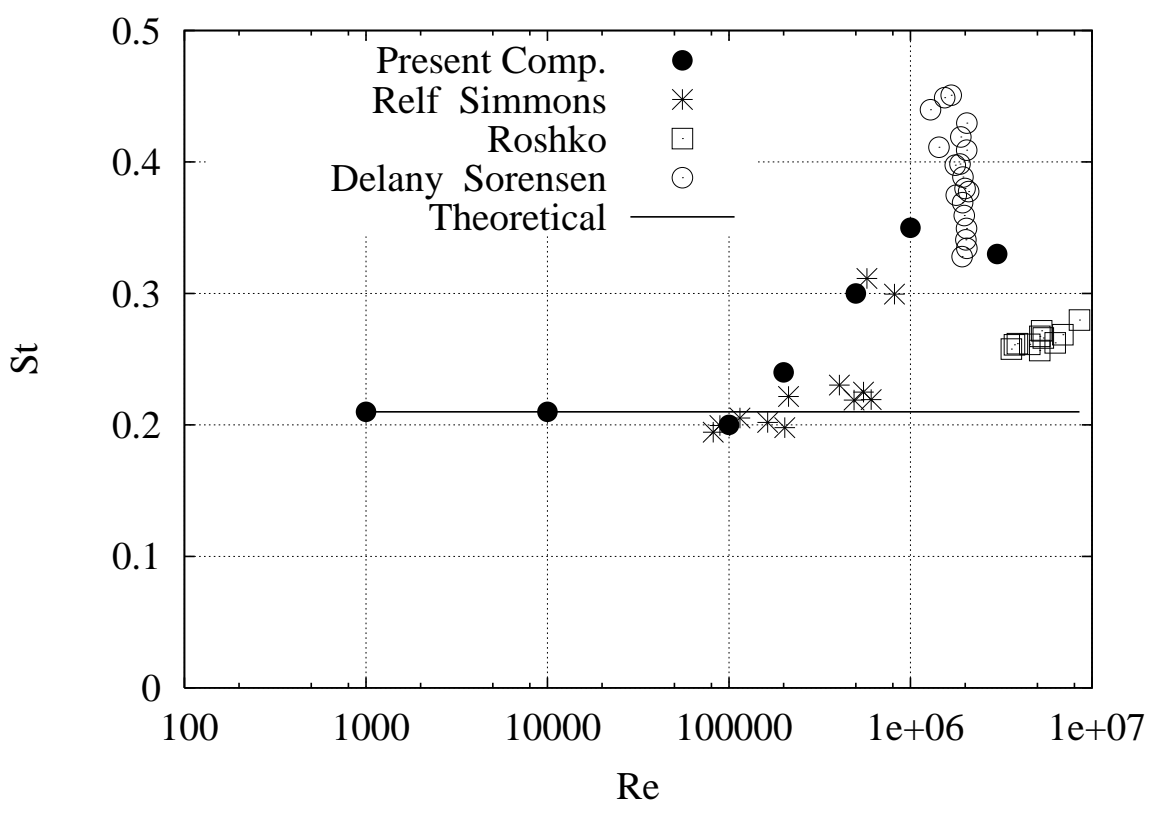

Figure 7. Variation of Strouhal number with with Reynolds number.

agreement with observations from experiments, and separates around 80 degrees and definitely before 90 degrees. At these high Reynolds numbers the wake becomes fully turbulent. A similar picture is observed at a Reynolds number of $2 \times 10^{5}$, but here the transition point is moving upstream closer to the separation line. For a Reynolds number of $5 \times 10^{5}$ the transition process takes place immediately right after the laminar separation, forcing the flow to reattach as turbulent flow, followed by turbulent separation further downstream. For the highest Reynolds number shown in Figure 9, the laminar separation immediately reattaches as turbulent flow, followed by a substantial region of attached flow before the flow finally has turbulent separation further downstream. The mechanism controlling the development of the drag crisis is clearly connected to the laminar turbulent transition process, interacting with the separation process and narrowing of the wake by delaying the separation as seen from the visualizations in Figure 8 and 10. Even though the overall behavior is the same for different experiments, there is a wide spread in the details of the flow, as shown by Achenbach [24] for the drag variation. These differences are caused by variation in the inflow turbulence, the surface roughness, and the aspect ratio of the cylinder among other parameters. Therefore, exact agreement should not be expected between computations and measurements either.

Table 1. The Strouhal number $\left(\frac{f \cdot D}{U}\right)$ number as function of Reynolds number.

\begin{tabular}{lllllll}
\hline$R e$ & $1 \times 10^{3}$ & $1 \times 10^{4}$ & $1 \times 10^{5}$ & $2 \times 10^{5}$ & $5 \times 10^{5}$ & $1 \times 10^{6}$ \\
St & 0.21 & 0.21 & 0.20 & 0.24 & 0.30 & 0.35 \\
\hline
\end{tabular}

\subsection{Discussion of Cylinder Results}

Generally, the code is capable of predicting the flow from the laminar region at a Reynolds number of 10 to fully turbulent/transitional flow at a Reynolds Number above one million. The use of the combined DES/transitional methodology improves the prediction of the drag crisis on the cylinder compared to pure DES simulation. Additionally, the DES/transitional methodology predicts the correct flow phenomena, with laminar separation, turbulent reattachment followed 
Separation point location

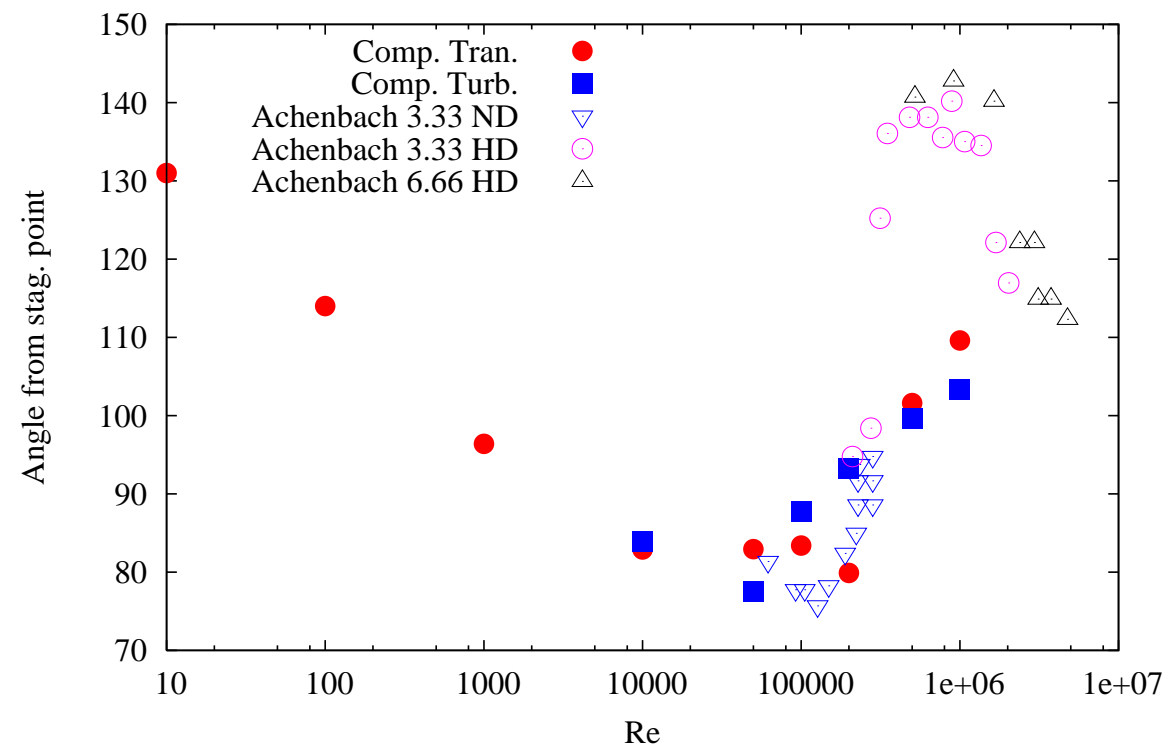

Figure 8. Computed averaged azimuth position of the wake separation point measured from the stagnation point, compare with data from [24]

by turbulent separation along with backward shift of the separation point and the narrowing of the cylinder wake with increasing Reynolds number.

\subsection{Flow over a thick airfoil}

Next, the method is applied to predicting the flow over the DU-96-W-351 airfoil, that has a thickness of $35 \%$ chord. Similar to the cylinder case, the separation of the flow over an airfoil is controlled by the momentum in the boundary layer flow and the skin friction distribution. With the good agreement obtained for the cylinder, it is of interest to investigate whether the transition/DES methodology can perform equally well for airfoil stall. As already addressed by Roshko [23], typical wall corrections used in wind tunnel experiments do not account for possible interference effects connected to flow separation which may be problematic for cases with a high degree of separation. Limited aspect ratio of two to four are often used in wind tunnels in connection with testing of airfoil sections due to the desire to obtain a high Reynolds number. When focusing on deep stall aerodynamics, this may work against the desire to limit the effects of the tunnel wall on the separation. The present data are kindly provided by LM Glasfiber, showing the stalling behavior of a thick DU-96 airfoil in the LM Glasfiber tunnel having a span to chord ratio of 1.5 .

Using the previously described computational mesh, the DU-96-W-351 airfoil was computed at a Reynolds Number of 3 million, with an aspect ratio (chord/span) of two using periodic conditions in the spanwise direction. Different values of inflow turbulence was investigated $[0.05 \%, 0.10 \%$ and $0.15 \%]$, in order to judge the importance of this parameter. A typical time trace of normal force is shown for the 15 degree angle of attack case for a period of $\sim 400$ dimensionless times, see Figure 11. For an airfoil with a chord of 0.9 meter and a velocity of $80 \mathrm{~m} / \mathrm{s}$ this would correspond to approximately 1.8 seconds; Eqn. 6 . Considering the highly unsteady signal, this must be considered a short averaging period, which will be reflected in the averaged pressure curves which do not fully collapse to a single curve for each considered case. The first series of computations were performed with the standard DES methodology, but these results showed a high dependency on the level of inflow turbulence, even at low angles of attack. This was identified as grid induced separation or MSD, and the application of the 


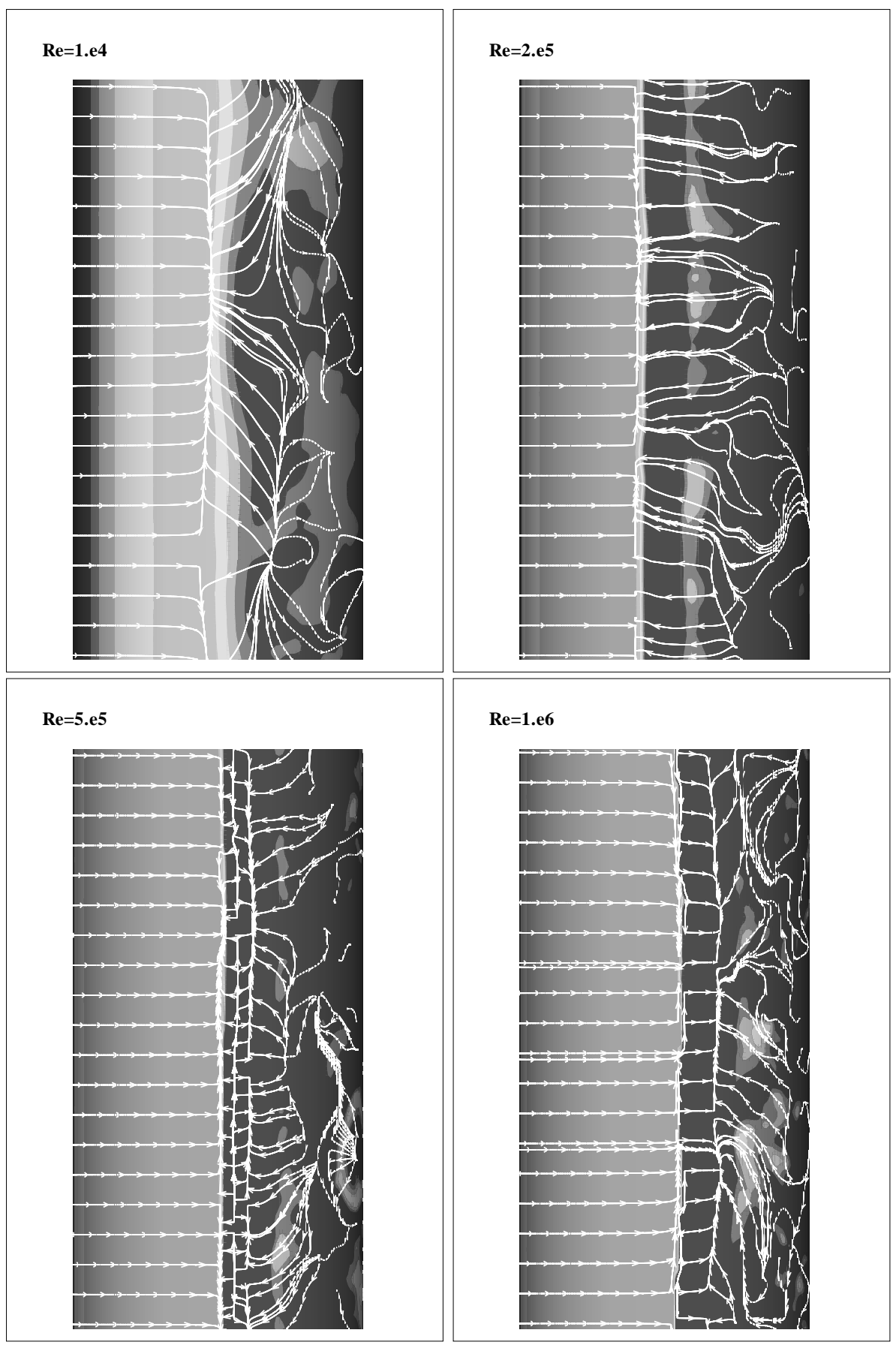

Figure 9. Instantaneous limiting streamlines on the surface of the cylinder for four cases of the simulations for transitional flow.

DDES methodology seems to alleviate this problem.

$$
t^{*}=\frac{\text { Chord }}{\text { Velocity }}
$$

Comparing the averaged lift and drag computed using the different turbulence intensities the following can be observed: At 10 degrees and below, the influence of the turbulence intensity is very limited. Right around the abrupt stall at 15 degrees, the computed results exhibit a large dependency on the inflow turbulence, showing a variation of nearly 20\%, see Figure 12 and 13. The turbulence intensity in the LM tunnel is reported to be around $0.1 \%$, but even small 


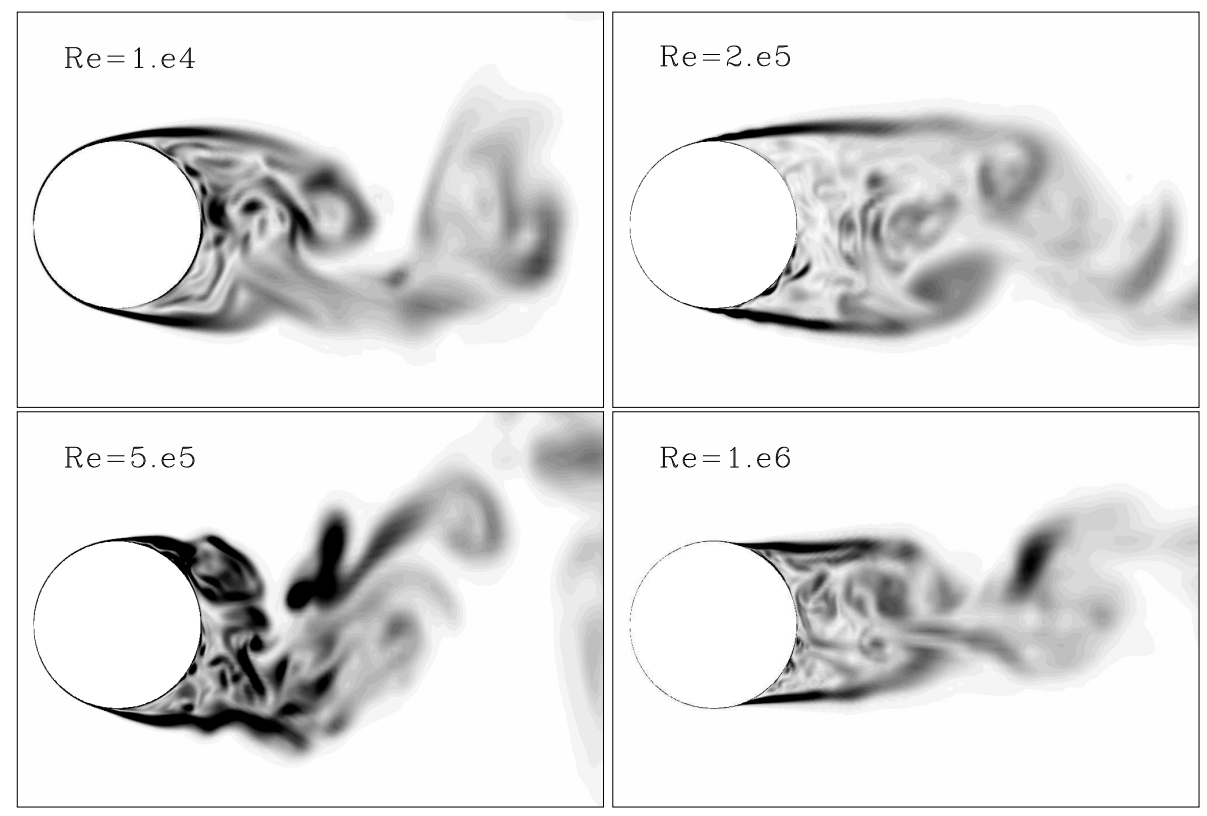

Figure 10. Representative snapshots of the absolute value of the vorticity behind the cylinder at four different Reynolds numbers, showing the narrowing of the wake with increasing Reynolds number.

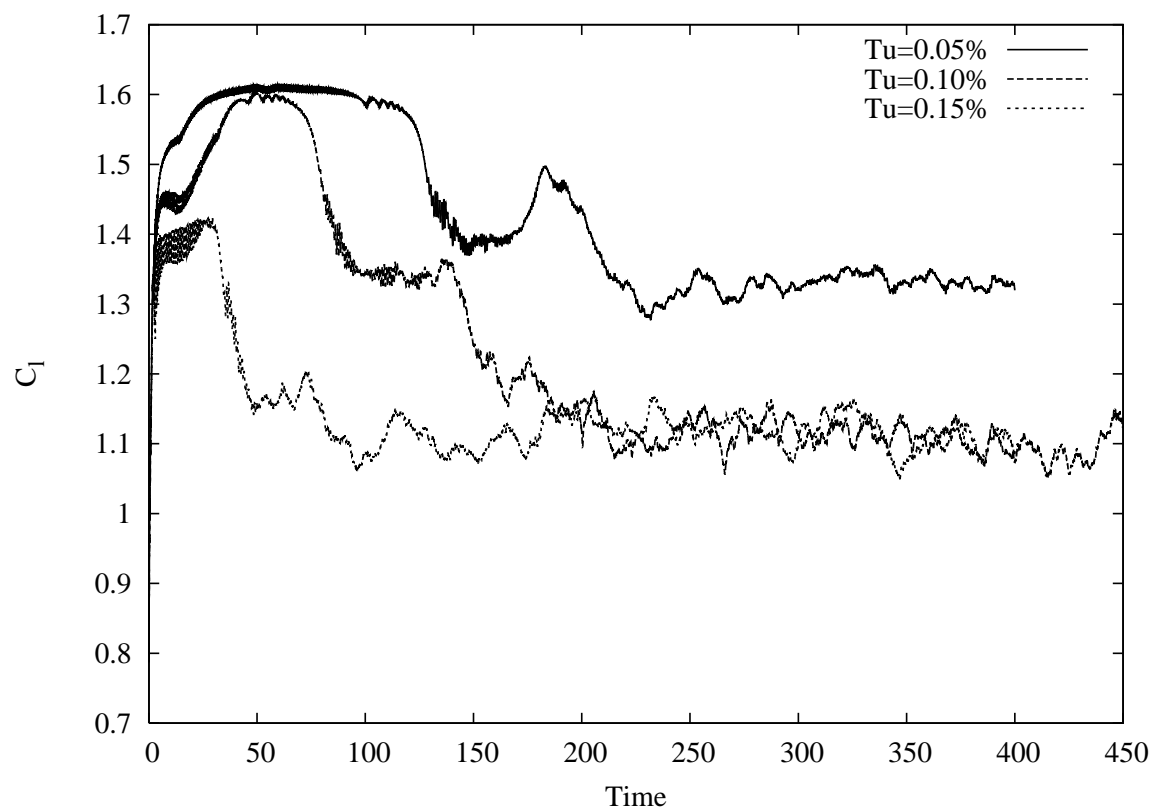

Figure 11. Time trace of computed normal force from a simulation at 15 degrees angle of attack for three different inflow turbulence intensities.

variations around this value in the computations give rather high variation right at stall. Comparing the computed 3D results using the Transition/DES methodology with measurements and standard 2D RANS transitional computations, we observe an increased accuracy of the 3D simulations.

Similar to the case of flow over a cylinder, the stalling characteristics of the thick DU-96-W351 airfoil is controlled by a balance between the momentum of the boundary layer flow and skin-friction at the wall. As a shift from laminar to turbulent flow will change the skin friction 


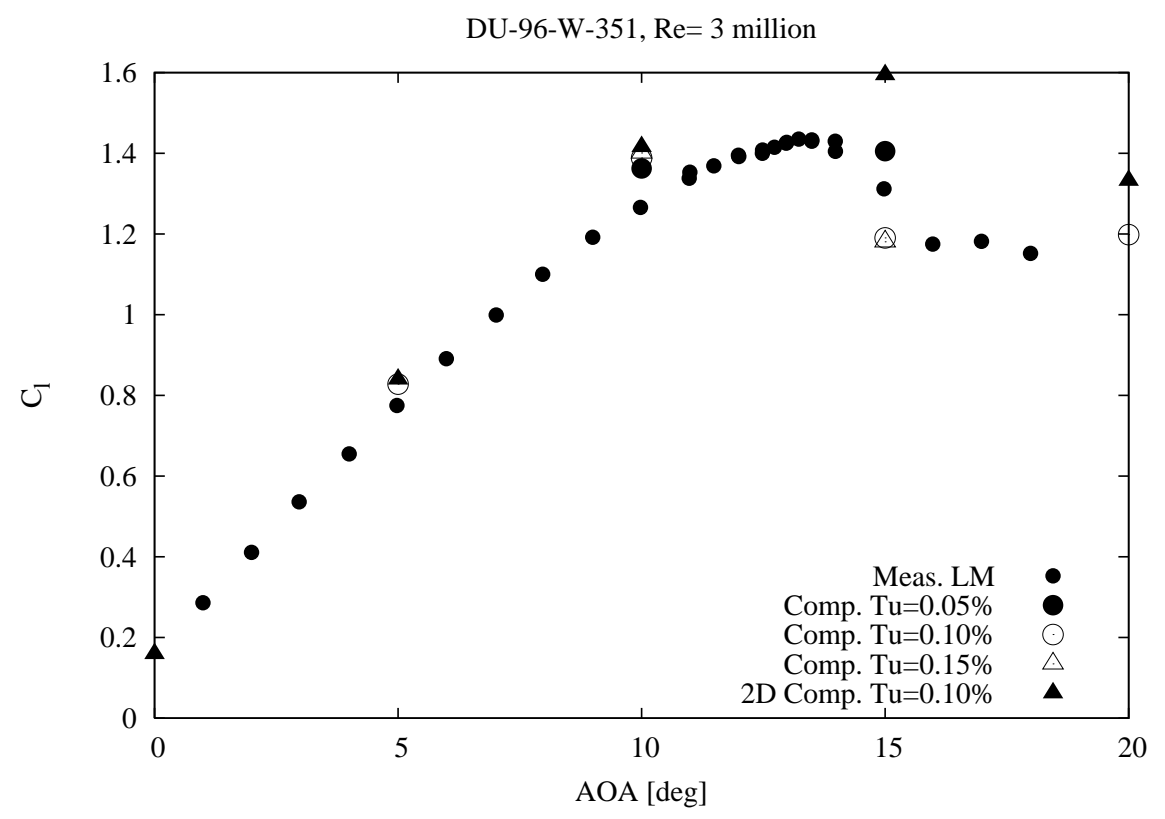

Figure 12. Comparison between computed lift at various turbulence intensities and measured values from the LM tunnel.

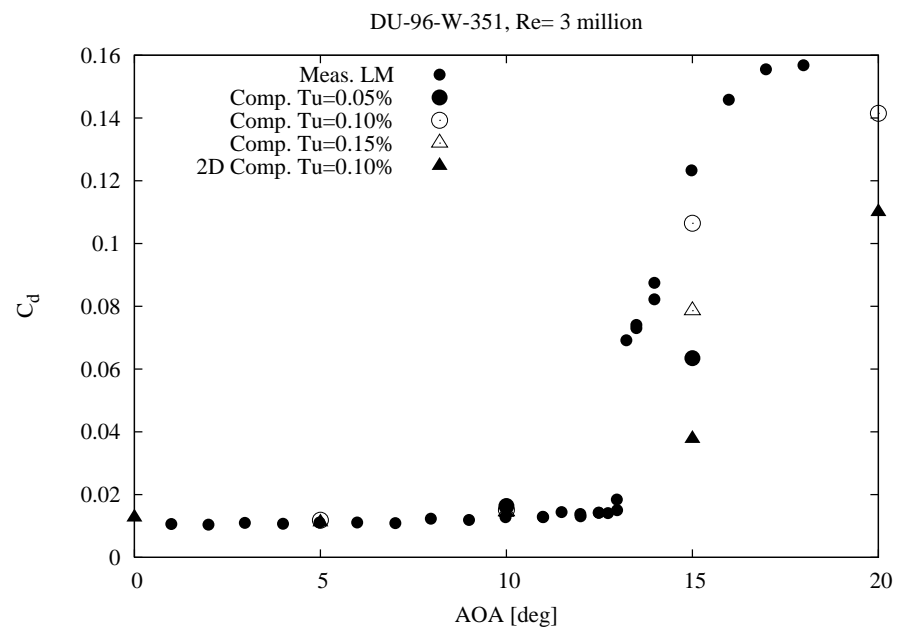

Figure 13. Comparison between computed drag at various turbulence intensities and measured values from the LM tunnel.

from a low to a high value, large changes in the separation pattern will result. The influence of the change to the inflow turbulence is clearly observed in Figure 15, where the best agreement is shown for a turbulence intensity of $0.1 \%$.

Similar to the cylinder analysis, the power spectrum of the tangential force for the DU-96$\mathrm{W}-351$ airfoil is analyzed to determine the Strouhal frequency of most energetic variations, performed for the case of 45 degrees angle of attack. As seen from Figures 6 and 16, the airfoil spectrum deviates from the cylinder spectrum in that high energy exists on frequencies lower than the shedding frequency. The Strouhal frequency can still be clearly identified at a frequency of 0.25 based on the airfoil chord. An alternative choice may be the projection of the chord on the direction perpendicular to the flow direction which would lower the frequency to around 71 percent of the previously mentioned value. 


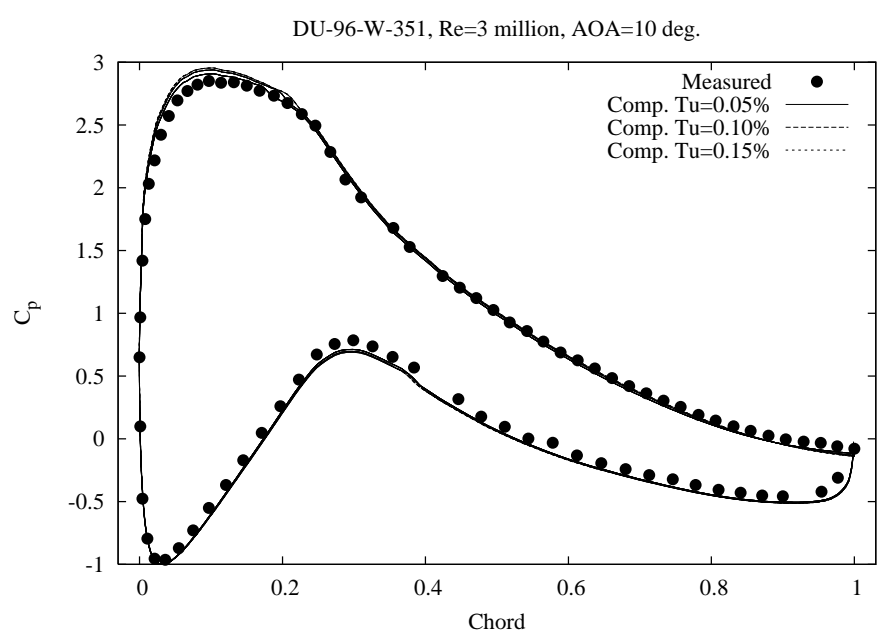

Figure 14. Pressure distribution around the DU-96-W-351 airfoil, comparison between computed values and measured distribution at 10 degrees.

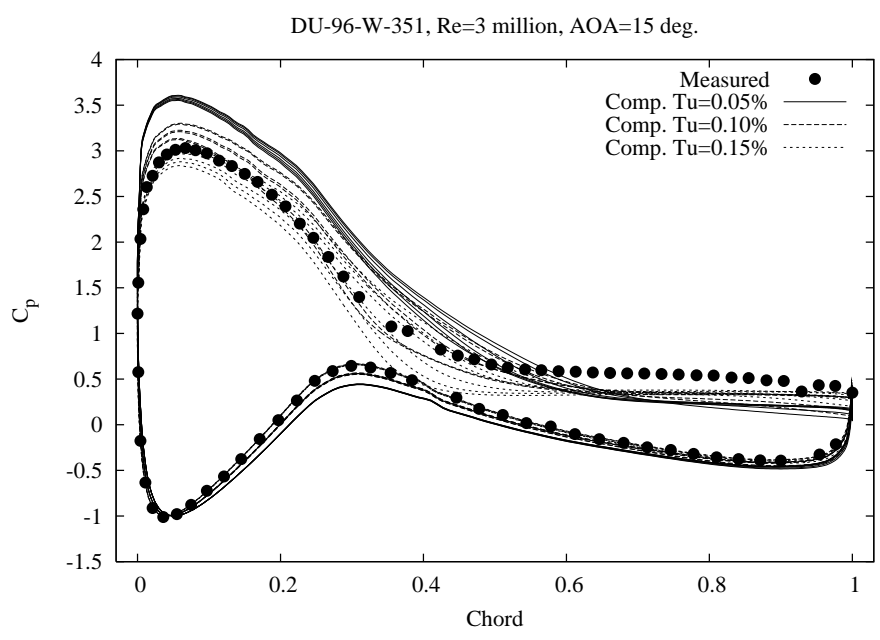

Figure 15. Comparison of pressure distributions around the DU-96-W-351 airfoil at 15 degrees for three different turbulence intensities. Averages at six spanwise positions are shown for each computation.

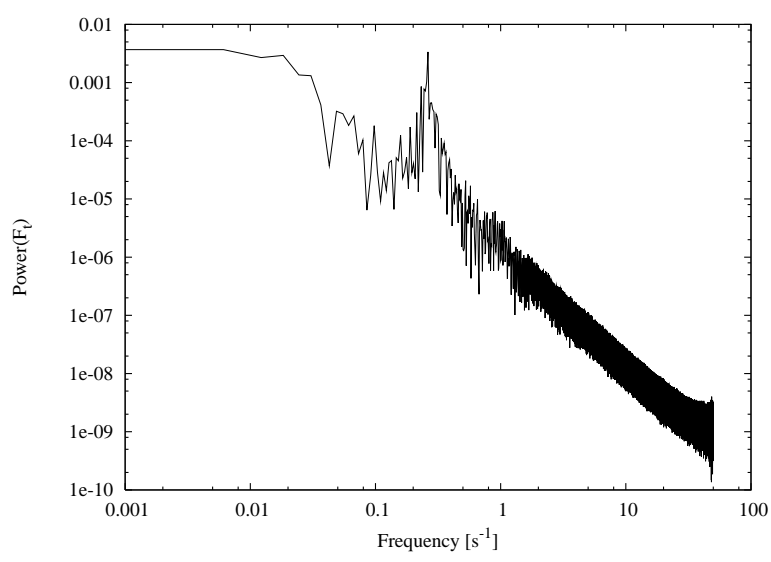

Figure 16. Power spectrum of the tangential force for the DU-96-W-351 airfoil at 45 degrees angle of attack, a clear Strouhal frequncy of $0.26 \mathrm{~Hz}$ is visible. 


\subsection{Discussion of Airfoil Results}

In the present work, computations of the flow over the DU-96-W-351, 35\% thick airfoil in the region of stalled flow have been compared to measurements from the LM tunnel. In the computations the airfoil section is modeled with an extent of two chords in the spanwise direction and the use of periodic conditions. This is done to limit the number of cells needed to model the actual configuration, where the airfoil section is enclosed by walls. Alternatively, symmetry conditions could be used at the two span-wise boundaries of the domain. This would prevent flow over these boundaries limiting the size of the turbulent structures in the separated area. This could be the focus of further work, along with efforts to actually resolve the wind tunnel walls. The problem of the wind tunnel corrections, that do not account for the interaction of the separation and the wall effects would additionally require further studies. The results show an improved agreement with the measured data, compared to 2D computations. Wether this increased accuracy will justify the much high increase in computational requirements from a few minutes for a 2D computations to several days for the 3D DES computations, will depend on the application.

\section{Conclusion}

The combination of Detached Eddy Simulations with a laminar to turbulent transition model has been demonstrated. The methodology has proven to be numerically very robust, and similar to the transitional computations using Reynolds Averaged Navier-Stokes approach, capable of predicting both laminar separation, turbulent reattachment, and turbulent separation. For the prediction of the cylinder drag crisis, a distinct improvement of the drag in the critical region is observed. For the airfoil the results are more inconclusive, but indicates that a very high dependency of the inflow turbulence intensity may exist for thick airfoils.

\section{Acknowledgement}

The work was funded by the Danish Energy Agency under contract ENS-33033-0055, Computations were made possible by the use of the MARY and Thyra PC-cluster at Ris $\varnothing$ National Laboratory and the DCSC, PC-cluster Yggdrasil and Alfheim. Measurement for the DU-96W-351 airfoil along with the airfoil contour was kindly provided by LM Glasfiber.

\section{References}

[1] F. R. Menter, R. B. Langtry, S. R. Likki, Y. B. Suzen, P. G. Huang, and S. Völker. A Correlation-Based Transition Model Using Local Variables, Part I - Model Formulation. In Proceedings of ASME Turbo Expo 2004, Power for Land, Sea, and Air, Vienna, Austria, June 14-17 2004. ASME. GT2004-53452.

[2] F. R. Menter, R. B. Langtry, S. R. Likki, Y. B. Suzen, P. G. Huang, and S. Völker. A correlation-based transition model using local variables, part ii - test cases and industrial applications. In Proceedings of ASME Turbo Expo 2004, Power for Land, Sea, and Air, Vienna, Austria, June 14-17 2004. ASME. GT2004-53454.

[3] M. Strelets. Detached Eddy Simulation of Massively Separated Flows. AIAA Paper 2001-0879, Russian Scientific Center "Applied Chemistry” St. Petersburg, 2001. 
[4] J. A. Michelsen. Basis3D - a Platform for Development of Multiblock PDE Solvers. Technical Report AFM 92-05, Technical University of Denmark, 1992.

[5] J. A. Michelsen. Block structured Multigrid solution of 2D and 3D elliptic PDE's. Technical Report AFM 94-06, Technical University of Denmark, 1994.

[6] N. N. Sørensen. General Purpose Flow Solver Applied to Flow over Hills. Risø-R- 827(EN), Risø National Laboratory, Roskilde, Denmark, June 1995.

[7] C. M. Rhie. A numerical study of the flow past an isolated airfoil with separation. PhD thesis, Univ. of Illinois, Urbana-Champaign, 1981.

[8] S. V. Patankar and D. B. Spalding. A Calculation Prodedure for Heat, Mass and Momentum Transfer in Three-Dimensional Parabolic Flows. Int. J. Heat Mass Transfer, 15:1787, 1972.

[9] S. V. Patankar. Numerical Heat Transfer and Fluid Flow. Hemisphere Publishing Corporation, 1980. ISBN: 0891165223.

[10] R. I. Issa. Solution of the Implicitly Discretised Fluid Flow Equations by OperatorSplitting. J. Computational Phys., 62:40-65, 1985.

[11] R. I. Issa, A. D. Gosman, and A. P. Watkins. The Computation of Compressible and Incompressible Recirculating Flows by a Non-iterative Implicit Scheme. J. Computational Phys., 62:66-82, 1986.

[12] P. K. Khosla and S. G. Rubin. A diagonally dominant second-order accurate implicit scheme. Computers Fluids, 2:207-209, 1974.

[13] F. R. Menter. Zonal Two Equation k- $\omega$ Turbulence Models for Aerodynamic Flows. AIAA-paper-932906, 1993.

[14] F.R. Menter and Kuntz M. The Aerodynamics of Heavy Vehicles: Trucks, Buses, and Trains, volume 19 of Lecture Notes in Applied and Computational Mechanics, chapter Adaptation of Eddy-Viscosity Turbulence Models to Unsteady Separated Flow Behind Vehicles, pages 339-352. Springer, 2004.

[15] N. N. Sørensen. CFD modeling of laminar-turbulent transition for airfoils and rotors using the gamma - Retheta model. In 2008 European Wind Energy Conference and Exhibition, , pages 106-112, Brussels (BE), 31 Mar - 3 Apr 2008 2008. EWEC.

[16] A. Toyoda, T. Misaka, and S. Obayashi. An Application of Local Correlation-Based Transition Model to JAXA High-Lift Configuration Model. AIAA Paper 2007-4286, June 2007.

[17] K. Pettersson and S. Crippa. Implementation and Verification of a Correlation Based Transition Prediction Method. AIAA Paper 2008-4401, 2008.

[18] R. B. Langtry, J. Gola, and F. R. Menter. Predicting 2D Airfoil and 3D Wind Turbine Rotor Performance using a Transition Model for General CFD Codes. AIAA-paper-20060395, 2006.

[19] N. N. Sørensen. HypGrid2D a 2-D Mesh Generator. Risø-R- 1035-(EN), Ris $\emptyset$ National Laboratory, Roskilde, Denmark, Feb 1998.

[20] C. Weiselsberger. New Data on The Laws of Fluid Resistance. AIAA Paper 84, NACA, 1922.

[21] E.F. Relf and L.F.G. Simmons. The frequency of eddies generated by the motion of circular cylinders through a fluid. Rep. and Mem. no. 917, Aero. Res. Counc., Lond., 1924.

[22] N.K. Delany and N.E. Sorensen. Low-speed drag of cylinders of various shapes. Tech. Note 3038, Nat. Adv. Comm. Aero., Wash., 1953. Note 3038. 
[23] A. Roshko. On the wake and drag of bluff bodies. J. Fluid Mech., year $=1961$, month $=$ , volume $=$, number $=10$, pages $=345-356$, note $=$,

[24] E. Achenbach. Distribution of local pressure and skin friction around a circular cylinder in cross-flow up to $R e=5 \times 10^{6}$. J. Fluid Mech., 34(4):625-639, 1968. 
Risø DTU is the National Laboratory for Sustainable Energy. Our research focuses on development of energy technologies and systems with minimal effect on climate, and contributes to innovation, education and policy. Risø has large experimental facilities and interdisciplinary research environments, and includes the national centre for nuclear technologies.

\section{Risø DTU}

National Laboratory for Sustainable Energy

Technical University of Denmark

Frederiksborgvej 399

PO Box 49

DK-4000 Roskilde

Denmark

Phone +45 46774677

Fax +4546775688

www.risoe.dtu.dk 